\title{
Effects of Electrophysiological Remodeling in Human Right Atrium: A Simulation Study
}

\author{
G Seemann ${ }^{1}$, H Ying $^{1}$, DL Weiss ${ }^{1}$, FB Sachse ${ }^{2}$, O Dössel ${ }^{1}$ \\ ${ }^{1}$ Institute of Biomedical Engineering, Universität Karlsruhe (TH), Germany \\ ${ }^{2}$ Nora Eccles Harrison Cardiovascular Research and Training Institute, University of Utah, USA
}

\begin{abstract}
Atrial fibrillation $(A F)$ induces variations in channel characteristics of human atrial myocytes. Data from experimental AF studies was incorporated in a computational model. It was used to investigate the effects of electrophysiological remodeling on myocardial behavior. This model was integrated in a schematic $2 D$ model of human right atrium including the sinoatrial node, crista terminalis, pectinate muscles, and the atrial working myocardium. Heterogeneous, anisotropic conduction was reconstructed with the monodomain approach.

In both normal and pathological case, the models were excited with 68 bpm by the sinoatrial node depolarizing spontaneously. A slightly decreased conduction velocity was reconstructed in the remodeling case. The repolarization period was abbreviated from physiologically $325 \mathrm{~ms}$ to $145 \mathrm{~ms}$. A shortened effective refractory period (ERP) and an attenuated ERP-rate relationship were identified. $B$ Both factors provide a substrate for $A F$ recurrence.
\end{abstract}

\section{Introduction}

Atrial fibrillation (AF) is an unphysiological rapid activation of the atria resulting in loss of contractility. The atrial rate normally given by the activation of the primary pacemaker, i.e. the sinoatrial node (SAN), with approximately 70 beats per minute (bpm) in rest is fastened to $400-600 \mathrm{bpm}$ due to e.g. re-entry mechanisms [1]. Potential consequences of chronic $\mathrm{AF}$ are e.g. severe congestive heart failure, thromboembolism and sroke, cardiomyopathy, and ventricular arrhythmia. Furthermore, clinical studies suggested that AF causes electrophysiological modifications, facilitating the progression of $\mathrm{AF}$ [2], i.e. electrophysiological remodeling [3]. The action potential duration (APD) in electrophysiological remodeled atrial tissue is very short already at slow rates associated with almost no further shortening for higher rates. Thus, AF induced remodeling increases the vulnerability to AF itself ("AF begets AF") [1].

\section{Materials and methods}

The experimental data of AF induced variations in ionic channel conductance and kinetics of human atrial myocytes reported in [4] consists of several electrophysiological changes. These changes have been incorporated in the Courtemanche-Ramirez-Nattel (CRN) [5] model to reproduce the behavior of human atrial myocytes of patients with chronic AF after cardioversion $[4,6]$.

In order to achieve this remodeling, several parameters were changed in the model: The conductance for $I_{K 1}$ was increased by $250 \%$. The pathological behavior for $I_{C a L}$ includes that the channel conductance was decreased by $74 \%$ and the time constant of the fast inactivation variable was increased by $62 \%$. The channel conductance for $I_{t o}$ was decreased by $85 \%$ and the activation curve was shifted by $+16 m V$. Finally, a shift of the inactivation curve of $I_{N a}$ by $+1.6 m V$ was reconstructed.

The resulting action potential (AP) of a remodeled cell arosen from the synergetic interaction of all abnormal configured currents is compared with the physiological AP in fig. 1. The simultaneous reduction in atrial $I_{t o}, I_{C a L}$ and increase in $I_{K 1}$ fully accounted for the acceleration of repolarization and the marked abbreviation of APD from

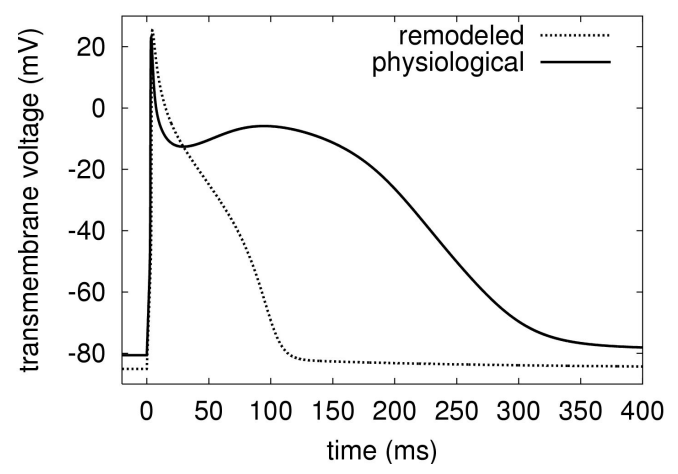

Figure 1. Simulated APs for a physiological cardiomyocyte and a cell with electrophysiological remodeling [6]. 
$305 \mathrm{~ms}$ to $112 \mathrm{~ms}$. The loss of plateau was partly due to the reduction in $I_{C a L}$. The enhanced $I_{K 1}$ contributed to the decrease of the resting potential from $-80 \mathrm{mV}$ to $-85 \mathrm{mV}$. The vanished notch in the remodeling case was caused by the reduced $I_{t o}$. Experimental recordings of AP in case of sinus rhythm and remodeling [7], in which the reduction of APD from $350 \mathrm{~ms}$ to $120 \mathrm{~ms}$ was reported, were comparable with the simulated results.

The electrophysiological remodeled description of the atrial working myocardium (AWM) was integrated in the geometrical model shown in fig. $2 \mathrm{~A}$. This anatomical model was a two-dimensional schematic representation of a part of the right atrium consisting of the heterogeneous SAN, fast conducting bundles, i.e. crista terminalis (CT) and pectinate muscles (PM), and the AWM itself. In contrast to the AWM, the other tissue classes were considered to be physiologic. In order to set the tissue specific electrophysiological properties of SAN, CT, and PM, the CRN model was adopted in a previous work [8]. The SAN model comprised central, transitional, and peripheral cells with different electrophysiological parameters. Central SAN cells developed a slower frequency of autorhythmic activity of 75 beats per minute (bpm) compared to the periphery with $86 \mathrm{bpm}$ [8]. The electrophysiological properties of the conduction bundles were changed based on measured data by adjusting maximum conductances of ion channels. Cells in the CT had a prolonged APD and an enlarged plateau voltage compared to the physiological AWM. The APD of PM cells was similar as in the original CRN model but the plateau voltage was lower [8].

Anisotropic coupling of cardiomyocytes was achieved with the monodomain model of electrical current flow. Transversal to longitudinal ratio of conductivity for the conduction bundles was set to 1:9 with the longitudinal direction being parallel to the main axis of the bundles. AWM and SAN were assumed to be isotropic in conduction. The transversal conductivity was set to $0.15 \mathrm{~S} / \mathrm{m}$ for AWM and CT, and to $0.11 S / m$ for PM. In the SAN, the electrical coupling between the cells was linearly reduced from peripheral $(0.11 \mathrm{~S} / \mathrm{m})$ towards central cells $(0.05 \mathrm{~S} / \mathrm{m})$ [8].

The effective refractory period (ERP) was determined in the schematic right atrial model by applying 5 beats with $1 \mathrm{~Hz}$ followed by one premature beat. ERP is defined as the shortest time at which the excitation from the premature beat was able to produce an excitation conduction [9].

\section{Results}

The activation sequence in the simulated schematic right atrium for the physiological case is shown in fig. 2 B-D. Due to the electrical interaction with adjacent tissue having a lower resting potential, the frequency of peripheral SAN cells was decreased and the activation started in central
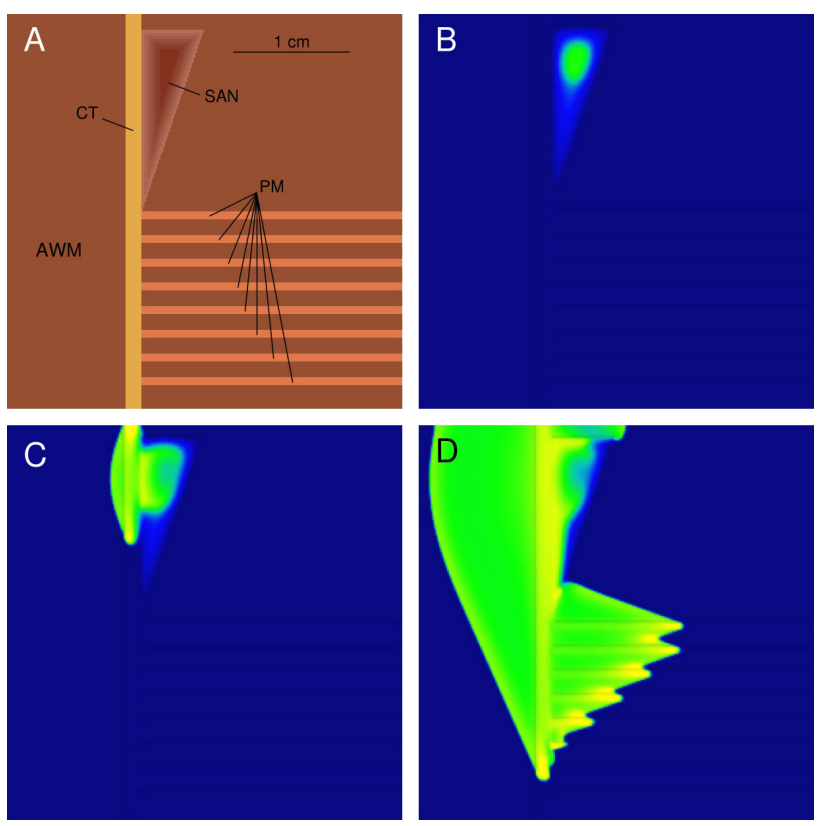

Figure 2. (A) Schematic model of a part of the right atrium. (B-D) Transmembrane voltage distribution during depolarization at (B) $100 \mathrm{~ms}$, (C) $200 \mathrm{~ms}$, and (D) $230 \mathrm{~ms}$ after begin of activation in the SAN center. Light color indicates $+10 \mathrm{mV}$, dark color $-80 \mathrm{mV}$.

SAN cells (fig. 2 B). Central SAN cells activated peripheral cells due to heterogeneous coupling in the SAN [10]. The depolarization was conducted preferentially from peripheral SAN cells into the CT (fig. 2 C) and from there into the PMs (fig. 2 D). Both conducting bundles were activating the AWM. The differences in activation sequence for the physiological model and the remodeling one were very small. Both models were excited by the SAN with a frequency of approximately $68 \mathrm{bpm}$. The activation time being the time to complete activation of the model was $73 \mathrm{~ms}$ in the physiological case and not significantly prolonged in the remodeling simulation. A slightly decreased conduction velocity (tab. 1) was simulated in the remodeling case presumably due to the $\mathrm{Na}^{+}$channel changes and the more negative resting potential.

Table 1. Simulated conduction velocity (CV) of different tissue types under physiological and remodeling condition. The differences are only due to changed electrophysiological parameters and not because of changed coupling.

\begin{tabular}{ccc}
\hline tissue & physiological $\mathrm{CV}(\mathrm{m} / \mathrm{s})$ & remodeling $\mathrm{CV}(\mathrm{m} / \mathrm{s})$ \\
\hline AWM & 0.53 & $0.5-0.51$ \\
CT & $1.18-1.2$ & $1.16-1.18$ \\
PM & $1.54-1.58$ & $1.52-1.56$ \\
\hline
\end{tabular}




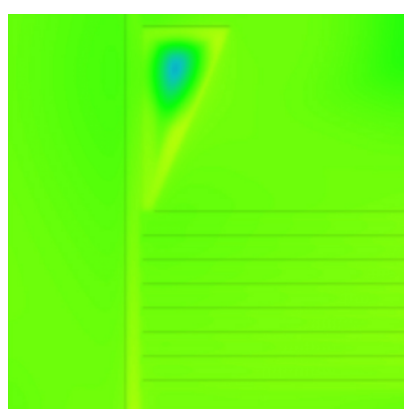

(a)

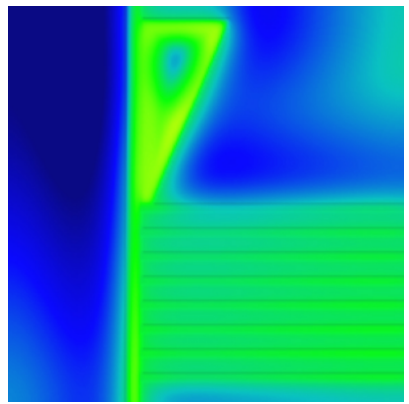

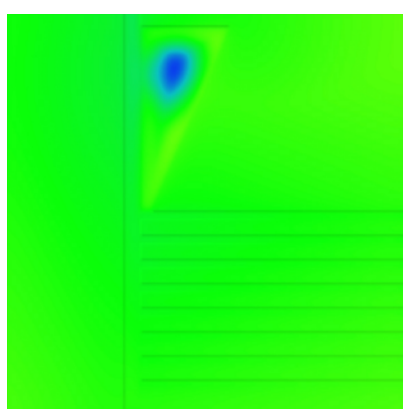

(b)

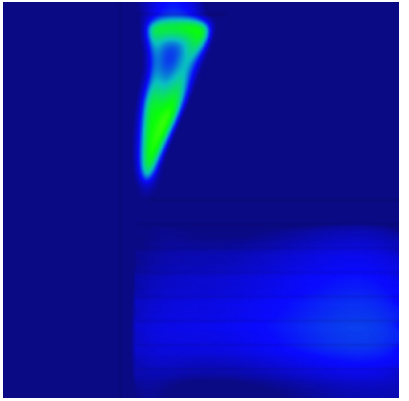

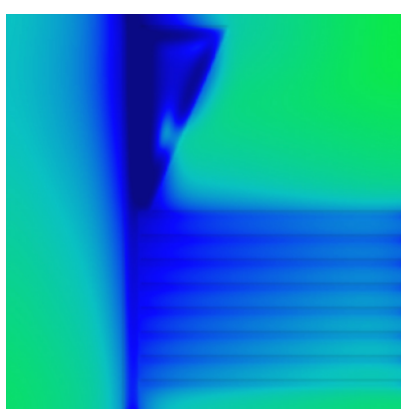

(c)

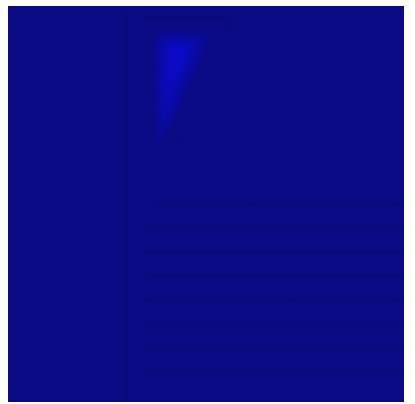

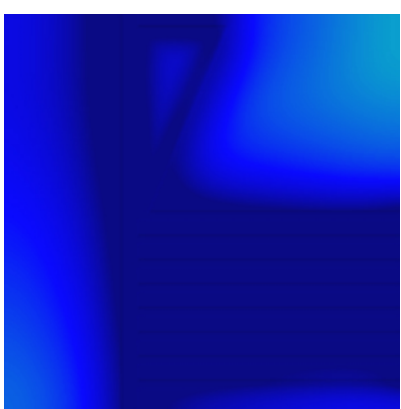

(d)

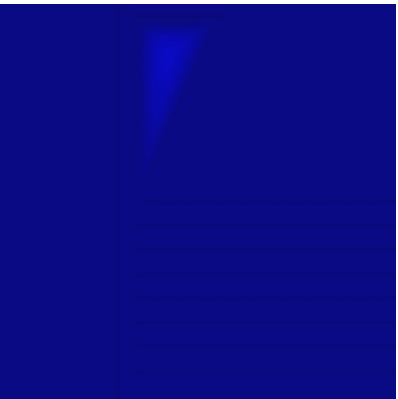

Figure 3. Progression of repolarization in the physiological (top) and electrophysiologically remodeled right atrium (bottom) [6]. Transmembrane voltage distribution is color coded as in fig. 2 at (a) $100 \mathrm{~ms}$, (b) $150 \mathrm{~ms}$, (c) $225 \mathrm{~ms}$, (d) $275 \mathrm{~ms}$ after activation of the CT.

Fig. 3 shows the repolarization process in both models. The significantly abbreviated time to complete repolarization reflects the shortening of the APD in the remodeled case. While the physiological right atrium was still in the plateau phase (fig. 3 a) the pathological started to repolarize beginning with AWM. When the repolarization process vanished in the remodeled AWM, CT, and PM, the physiological model just started to repolarize in the SAN center (fig. $3 \mathrm{~b}$ ). The physiological right atrium began to repolarize, as the entire repolarization process was finally proceeded in the remodeling case (fig. $3 \mathrm{c}$ ). The repolarization was more homogeneous in the physiological model. The AWM in the remodeling case was completely repolarized after $145 \mathrm{~ms}$. The physiological model repolarized completely after $325 \mathrm{~ms}$ (fig. $3 \mathrm{~d}$ ).

Critical components for patients with chronic AF were the markedly abbreviated ERP and the attenuated ERP rate dependency, which were also observed in patients after cardioversion [11, 12]. Fig. 4 depicts for the simulations the remarkably abbreviated ERP at any cycle length, and the attenuated ERP rate dependency in the remodeling case. As the cycle length increases from 200 to $600 \mathrm{~ms}$, the slope of ERP restitution in the physiological case is much steeper than in the remodeling case. The ERP is largely influenced by the duration of final repolarization of cells in the pacing site and the electric coupling to surrounding tissue [13].

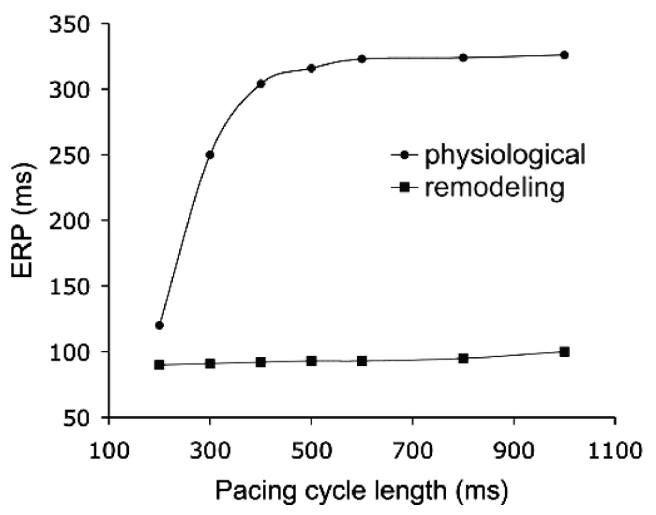

Figure 4. Effective refractory period of AWM in control (circles) and chronic AF group (squares) plotted against various cycle lengths [6].

\section{Discussion and conclusions}

A short refractory period and its small adaptation to heart rate are viewed as the electrophysiological milieu for AF recurrence, which results in a shorter wavelength of the atrial activity. This allows more multiple circuits coexisting in the given tissue, and the vulnerability for $\mathrm{AF}$ increases. AF recurs in patients after cardioversion, if the triggers, e.g. ectopic beats, encounters in the 
atrium. Chronic AF is associated with electrophysiological changes, which induce the pronounced reduction of the ERP. Thus, the decreased wavelength is determined. The alteration facilitates the maintenance and perpetuation of AF. This mechanism of "AF begets AF" was reconstructed in the simulations.

The simulated APDs and ERPs for the normal and remodeled case were consistent with experimental findings $[7,14]$. The same heart rate in the physiological and pathological case with $68 \mathrm{bpm}$ was not expected. The frequency of the sinoatrial node (SAN) should decrease due to the coupling to the AWM with more negative resting potential in the remodeled case. This disadvantage of the model can be overcome, if the remodeling condition is incorporated not only in the AWM, but also in CT and PMs. Since the CT was the first tissue to be activated (fig. 2 C) and directly joined with the SAN, the remodeling in CT will have a great impact on the frequency of the SAN.

The slightly decreased conduction velocity (CV) shown in tab. 1 indicates that changed electrophysiological properties were unessential factors to induce the measured decreased $\mathrm{CV}[11,12]$. This reduction is occurring in AF patients after cardioversion. A reduced $I_{N a}$ is able to decrease the steepness of the upstroke in depolarization phase. But the altered $I_{N a}$ in the remodeling case had only small influence on the AP. Thus, in this simulation the decrease in $\mathrm{CV}$ in AWM was explained by the more negative resting potential. Therefore, it takes longer for the myocytes to reach the threshold and to depolarize. Another factor determining the $\mathrm{CV}$ is the coupling. In several studies, the effects of AF on the expression of atrial gap junctions influencing myocardial conduction were investigated [7]. A reduced electrical coupling between cardiomyocytes through gap junctions were considered as essential factors for the decreased CV $[11,15]$. In future work, the reduced electrical coupling between cardiomyocytes during electrophysiological remodeling needs to be incorporated in detail.

In summary, the model reconstructed mechanisms of electrophysiological remodeling caused by AF in tissue. In future, effects of other pathologies like mutations in atrial cells [16] on the behavior of the right atrium will be investigated. The model will be used to verify the mechanisms of AF initiation and perpetuation and will be enhanced by considering realistic atrial shape in order to understand the influence of individual geometry on atrial activity.

\section{References}

[1] Nattel S. New ideas about atrial fibrillation 50 years on. Nature 2002;415:219-226.

[2] Wijffels MCEF, Kirchhof CJHJ, Dorland R, Allessie MA. Atrial fibrillation begets atrial fibrillation. Circ 1995; 92:1954-1968.
[3] Van Wagoner DR. Electrophysiological remodeling in human atrial fibrillation. Pacing Clin Electrophysiol 2003; 26(7-II):1572-1575.

[4] Zhang H, Zhu JJ, Garratt GJ, Holden AV. Cellular modelling of electrical remodelling in two different models of human atrial myocytes. In Proc. Computers in Cardiology, volume 30. 2003; 777-780.

[5] Courtemanche M, Ramirez RJ, Nattel S. Ionic mechanisms underlying human atrial action potential properties: Insights from a mathematical model. Am J Physiol 1998; 275(44):H301-H321.

[6] Ying H. Investigation of atrial fibrillation induced remodeling in human right atrium: A simulation study, Universität Karlsruhe (TH), Institut für Biomedizinische Technik 2004. Student Research Project.

[7] Bosch RF, Zeng XR, Grammer JB, Popvic K, Mewis C. Ionic mechanisms of electrical remodeling in human atrial fibrillation. Cardiovasc Res 1999;44:121-131.

[8] Seemann G, Höper C, Sachse FB, Dössel O, Holden AV, Zhang H. 3D anatomical and electrophysiological model of human sinoatrial node and atria. Phil Trans Roy Soc 2006; Accepted.

[9] Tse HF, Lau CP, Ayers GM. Heterogeneous changes in electrophysiologic properties in the paroxysmal and chronically fibrillating human atrium. J Cardiovasc Electrophysiol 1999;10:125-135.

[10] Seemann G, Weiß DL, Sachse FB, Dössel O. Interaction of the human sinoatrial node with surrounding atrial tissue: A simulation study. Biomedizinische Technik 2005; 50-1:560-561.

[11] Raitt MH, Kusumoto W, Giraud GD. Electrophysiologic predictors of the recurrence of persistent atrial fibrillation within 30 days of cardioversion. Am J Cardiol 2004; 93:107-110.

[12] Allessie MA, Boyden PA, Camm AJ. Pathophysiology and prevention of atrial fibrillation. Circ 2001;103:769-777.

[13] Workman AJ, Kane KA, Rankin AC. The contribution of ionic currents to changes in refractoriness of human atrial myocytes associated with chronic atrial fibrillation. Cardiovasc Res 2001;52:226-235.

[14] Yu WC, Lee SH, Tai CT. Reversal of atrial electrical remodeling following cardioversion of long-standing atrial fibrillation in man. Cardiovasc Res 1999;42:470-476.

[15] van der Velden HM, Jongsma HJ. Cardiac gap junction and connexins: Their role in atrial fibrillation and potential as therapeutic targets. Cardiovasc Res 2002;54(2):270-279.

[16] Seemann G, Weiß DL, Sachse FB, Dössel O. Simulation of the long-QT syndrome in a model of human myocardium. Computers in Cardiology 2003;30:287-290.

Address for correspondence:

Dr.-Ing. Gunnar Seemann

Institut für Biomedizinische Technik

Universität Karlsruhe (TH)

Kaiserstr. 12 / 76128 Karlsruhe / Germany

tel./fax: ++49-721-608-2790/2789

Gunnar.Seemann@ibt.uni-karlsruhe.de 